\title{
High Grade Prostatic Intraepithelial Neoplasia and the Risk of Prostate Cancer
}

\author{
Gregory P. Swanson, A. Taylor Kingman, Courtney N. Shaver, Yolanda Munoz Maldonado, \\ T. Phillip Reilly \\ Baylor Scott and White Health Care, Temple, Texas, USA \\ Email: gregory.swanson@BSWHealth.org
}

How to cite this paper: Swanson, G.P., Kingman, A.T., Shaver, C.N., Maldonado, Y.M. and Reilly, T.P. (2018) High Grade Prostatic Intraepithelial Neoplasia and the Risk of Prostate Cancer. Open Journal of Urology, 8, 67-76.

https://doi.org/10.4236/oju.2018.83008

Received: March 6, 2018

Accepted: March 27, 2018

Published: March 30, 2018

Copyright $\odot 2018$ by authors and Scientific Research Publishing Inc. This work is licensed under the Creative Commons Attribution International License (CC BY 4.0).

http://creativecommons.org/licenses/by/4.0/

(c) (i) Open Access

\begin{abstract}
Introduction: High Grade Prostatic Intraepithelial Neoplasia (HGPIN) was originally thought to be a cancer precursor, but subsequent data has questioned its prognostic significance. We analyzed a large cohort of men diagnosed with HGPIN for subsequent occurrence of prostate cancer. Methods: From 2001 to 2011, we identified 567 men with isolated HGPIN and followed them for subsequent diagnosis of prostate cancer. Results: Two hundred and five patients were followed (median 5.9 years) without biopsy and remained clinically free of prostate cancer. The remaining 362 men underwent repeat biopsies and 133 (37\%) were diagnosed with prostate cancer. The number of cores of HGPIN and whether they were unilateral or bilateral was not predictive for subsequent diagnosis of cancer. Prostate specific antigen was the only statistically significant predictor for prostate cancer. Conclusions: We found the incidence of cancer after a diagnosis of HGPIN to be $37 \%$, which is consistent with other published series. This is only marginally higher than in patients re-biopsied after a prior benign biopsy. It appears that isolated HGPIN has only a small predictive value for subsequent diagnosis of prostate cancer. Therefore the finding of HGPIN should be used only in conjunction with other risk factors and patient considerations in deciding whether to proceed with further prostate biopsies.
\end{abstract}

\section{Keywords}

HG PIN, Prostate Biopsy, Prostate Cancer, Prostate Cancer Risk

\section{Introduction}

Prostatic intraepithelial neoplasia (PIN) consists of atypical cells with the appearance of neoplasia within prostate acini or ducts, but with minimally or 
non-disrupted basement membrane (basal layer): i.e. no evidence of invasion. The abnormal cells share immunohistochemical, morphologic, and genetic changes with cancer. With its acceptance as a distinct entity in the 1980s, some have considered PIN to be a predecessor to overt prostate cancer-especially if it is high grade PIN (HGPIN) [1] [2].

High grade PIN is a not uncommon, with a mean incidence in more contemporary data of $7.7 \%$ (range from $0 \%$ to $24.6 \%$ ) of all prostatic biopsies [3] [4]. The subsequent diagnosis of prostate cancer in those patients is $21 \%$ to $48 \%$, demonstrating less than complete penetrance [5]-[10]. In a review of multiple studies of HGPIN patients from 1991 to 2005, the average chance of finding cancer on subsequent biopsy was $32 \%$. Studies with larger number of patients ( $>50)$ generally have a lower incidence $(\sim 25 \%)$ [3].

As a result of the perceived association between the HGPIN and prostate cancer, some have recommended an aggressive re-biopsy schedule to detect concomitant or subsequent cancer in patients with HGPIN [11] [12]. More recently, some have suggested that with obtaining more cores than done historically [13], there is less likelihood of finding cancer on subsequent biopsy in men with HGPIN, so HGPIN should not be the primary driver for re-biopsy [1]. Concomitant to that, the European Association of Urology guidelines on prostate cancer state that HGPIN alone is no longer considered an indication for repeat biopsy [14]. It would appear that the significance of HGPIN and its implications remains uncertain. We looked at our own experience in a large population to try to understand the significance of HGPIN on the risk of prostate cancer.

\section{Methods}

After institutional review board approval, a retrospective review of our pathology database (which archives all the pathology reports in a computer database) from 2001 to 2011 was performed to identify all patients who underwent transrectal ultrasound guided prostate biopsy. A total of 6101 prostate biopsies were identified. There were 650 patients with isolated HGPIN (no cancer) on needle biopsy. Some of these patients (82) also had atypical small acinar proliferation (ASAP) on the HGPIN biopsy and initially were included. All patients with concomitant cancer or previous abnormal biopsies were excluded. HGPIN was diagnosed on microscopic examination by the presence of cytologically malignant nuclei (vesicular, with prominent nucleoli) seen in prostatic epithelial cells without evidence of invasion. Transrectal biopsies were done, with the number of cores taken at the urologists discretion. Specimens were fixed in formalin and processed routinely by the institution's pathology department. After identifying these patients, a comprehensive review of the electronic medical record was used to identify demographics, including the following criteria: age, number of cores taken, Prostate specific antigen (PSA), prostate volume, number of repeat biopsies, number of cores of HGPIN, laterality (unilateral vs. bilateral) of HGPIN, incidence of subsequent prostate cancer, and final Gleason score (patients who 
developed prostate cancer). With the preponderance of referrals coming because of an elevated PSA, the recording of findings on digital rectal exam was imprecise and incomplete, so was not thought to be a reliable metric. All patient data was collected and entered into a spreadsheet that was then utilized for biostatististical analysis.

\section{Results}

Six hundred fifty men were identified with HGPIN without obvious cancer. Eighty-two patients also had ASAP, which on univariate analysis was significantly associated $(p<0.0001$, OR $2.7[1.6-4.3])$ with the subsequent diagnosis of cancer. They were removed from further analysis to avoid confounding the results of HGPIN. This left 568 patients with HGPIN only. Table 1 shows the patient characteristics. The median age was 68 years. The majority of the patients $(362,64 \%)$ underwent at least one subsequent biopsy. Of those that did not undergo a subsequent biopsy, the vast majority (86\%) were followed more than 2 years (clinical follow up). In these patients, a repeat biopsy was not considered clinically indicated. Of those that underwent subsequent biopsy, the time to second biopsy was a median of 7 months, with $78 \%$ occurring within two years. Figure 1 shows the patient flow.

In considering only those patients that underwent repeat biopsy $(n=362)$, $37 \%$ were diagnosed with cancer. Of those diagnosed with cancer, $58 \%$ were found on the first post HGPIN biopsy, while the remaining patients were found on subsequent biopsies.

For the index biopsy (the first one containing HGPIN), the median number of cores taken was 13 (range 3 - 55; first quartile 11, fourth quartile 18). The median PSA was $5.40 \mathrm{ng} / \mathrm{ml}$ (quartiles 4.10, 7.70, mean 6.54). PSA density (PSAD) was a median of 0.11. HGPIN was unilateral in $417(73 \%)$ and bilateral in 151 (27\%). Unilateral vs bilateral (OR 1.098 CI $0.710-1.698, p=0.6751)$ and single

Table 1. Patient characteristics.

\begin{tabular}{cccc}
\hline & All 568 pts & $\begin{array}{c}\text { Subsequent } \\
\text { biopsy pts }\end{array}$ & No biopsy pts \\
\hline Median age (years) & 68.0 & 67.7 & 68.2 \\
Cores taken & $13(1-132)$ & $18(1-132)$ (re-biopsy) & - \\
Median PSA (ng/ml) & 5.40 & 5.70 & 5.0 \\
PSAD & 0.14 & 0.18 & 0.10 \\
\% Unilateral HGPIN & $73 \%$ & $73 \%$ & $75 \%$ \\
\% Bilateral HGPIN & $27 \%$ & $27 \%$ & $25 \%$ \\
\% Single core & $53 \%$ & $50 \%$ & $59 \%$ \\
\% multiple cores & $47 \%$ & $50 \%$ & $41 \%$ \\
$\begin{array}{c}\text { Subsequent diagnosis } \\
\text { with cancer }\end{array}$ & $24 \%$ & $37 \%$ & - \\
\hline
\end{tabular}

Pts $=$ patients. PSAD = PSA density. HGPIN = high grade prostatic intraepithelial neoplasia. 


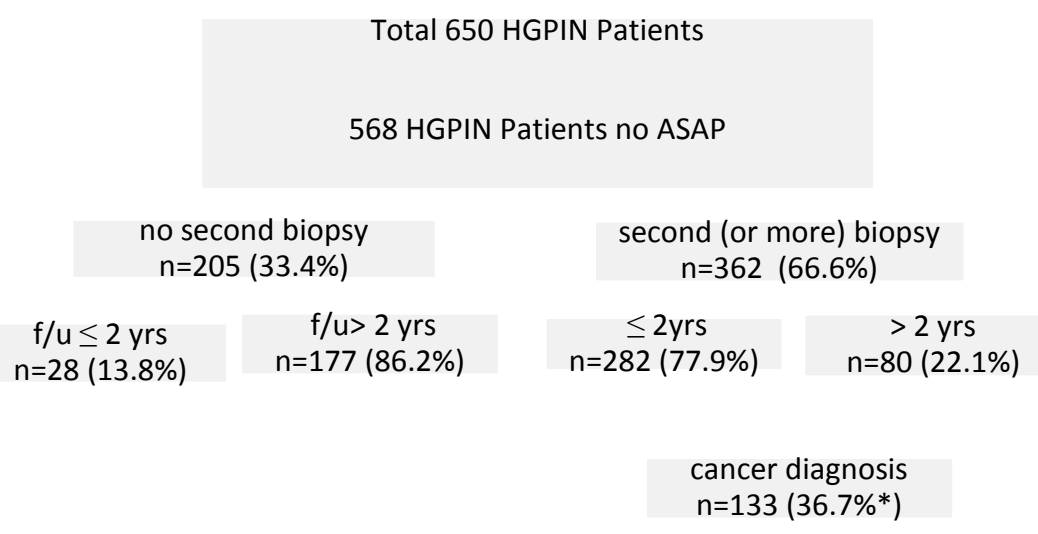

Figure 1. Patient disposition $\left(\mathrm{f} / \mathrm{u}=\right.$ follow up). ${ }^{\star}$ of re-biopsy patients $23.4 \%$ of total patients.

vs multiple cores (OR 1.065 CI $0.721-1.572, p=0.7523$ ) of HGPIN were not statistically significant predictors for subsequent diagnosis of cancer. For those with a second biopsy, the PSA median was $5.70 \mathrm{ng} / \mathrm{ml}$ (quartiles 3.5, 8.4, mean 7.54). PSAD was a median of 0.12. Both the PSA and PSAD were slightly higher than in the original cohort. For the second biopsy, the median number of cores taken was 18 (range 1 - 132, quartiles 12, 20), also higher than baseline. For those clinically followed without PSA, the median PSA at index biopsy was 5.0 (quartiles 3.4; 7.30, mean 5.85) with a PSAD of median 0.10, both lower than the entire cohort and certainly lower than those that underwent re-biopsy. For those followed clinically, the median follow up was 5.9 years.

For patients with a positive biopsy for cancer the median PSA was $9.1 \mathrm{ng} / \mathrm{ml}$ (range 0.4 - 217.1, quartiles 5.6; 15.8, mean 13.93.) For those that had a negative biopsy the median PSA was $5.1 \mathrm{ng} / \mathrm{ml}$ (range 0.2 - 56.3, quartiles 3.0; 7.7, mean $6.25)$.

The grade for those diagnosed with cancer was Gleason 6 in 49.2\%, Gleason 7 in $40 \%$ and Gleason 8 and 9 in 8\%; $20 \%$ had primary Gleason 4 with only 2 patients with primary Gleason 5 cancer.

Evaluating the factors of age, baseline PSA, PSAD, unilateral vs bilateral HGPIN, and single core vs multicore HGPIN-only PSA $(p=0.0002)$ and PSAD $(p \leq 0.001)$ were significate in the univariate logistic regression with cancer diagnosis as the outcome. A multivariate logistic regression model using the same 5 covariates used in the univariate analysis and cancer diagnosis as the response variable was fit. Model selection methods were used and all included only PSA in the model $(p=0.0002)$. The $c$-statistic of 0.611 indicated that this model with only baseline PSA would predict slightly better than chance. A receiver operator curve using this model demonstrated three potential cutpoints for PSA, 6.1, 4.8 and $2.8 \mathrm{ng} / \mathrm{ml}$, but the area under the curve was no better than 0.59 for any one of them, with a positive predictive value no better than $42.8 \%$ for any one of them. Just looking at PSA levels, for those with a baseline PSA of $<5 \mathrm{ng} / \mathrm{ml}, 26 \%$ were subsequently diagnosed with cancer, for PSA $5-10 \mathrm{ng} / \mathrm{ml}, 39 \%$ and for 
PSA $>10 \mathrm{ng} / \mathrm{ml}, 55 \%$. A chi square test showed that there was a significant difference in the distribution of patients with or without cancer between these three PSA groups $(p=0.0007)$.

\section{Discussion}

This data represents one of the largest single institution reports on the finding of HGPIN with subsequent re-biopsy. As with most reports, there is no requirement that all patients are re-biopsied, and follow-up generally is limited. The lack of a standard protocol for the management of HGPIN makes it difficult to precisely determine the indications for subsequent biopsy and the determination of the exact risk of HGPIN for the subsequent detection of prostate cancer. As we evaluated our data and compared it with published results, it is apparent universally the selection bias is high. For example, from the literature, follow-up biopsy of patients with a finding of HGPIN does not appear to be the routine, generally under $50 \%$ (46\% [12], 45\% [15], 27\% (year one) [16]). In our series, almost $2 / 3$ underwent re-biopsy.

We observed that $37 \%$ of our patients were found to have prostate cancer on re-biopsy. The majority of those biopsies occurred within two years (median 7 months). The median number of cores increased from 13 on the baseline biopsy to 18 on the re-biopsy. The rapidity of re-biopsy and the increase in the number of cores would indicate that in those patients, the finding of HGPIN was concerning, prompting re-intervention. The positive rate is comparable to some of the larger studies with an overall positive biopsy rate similar to our $37 \%$ (broadly $23 \%-44 \%$ but mostly in the $32 \%-36 \%$ range [11] [12] [17] [18]). It is noted that when biopsies are restricted to the first year after the finding of HGPIN, the cancer rate is lower at $18 \%-23 \%$ [9] [16] [19].

The detection of cancer on re-biopsy is trending downward. This has been attributed to more aggressive biopsy techniques initially, with more cancers being detected up front [1]. Specifically, even a small shift from 6 to 8 cores to $8+$ cores on the initial biopsy reduces the subsequent finding of cancer [9]. By the year 2000 , there was a concerted change from 6 core sextant biopsies to 10 or 12 cores [13].

There have been efforts to improve the predicative power of HGPIN. The information available usually includes age, number of cores, PSA, PSA density, PSA velocity, number of cores involved with HGPIN, and whether there was unilateral vs bilateral involvement. However, not all these parameters are available in every series and the results are not consistent. It is also notable that the pathology is not always clear. In a study with patients from the 1990s, of 346 patients with a diagnosis of HGPIN, 101 of them were eliminated on pathology review [17]. This is from an institution noted for their genitourinary pathology, which speaks to the subjectivity of pathologic diagnosis. Subsequently, the same institution reported on 791 men with isolated HGPIN, all of whom had a second biopsy within a year [9]. It was not reported as to how many other patients had 
high grade PIN and were not biopsied. However, of the 791 men, 18\% had cancer, and with their selection factors, neither PSA nor number of HGPIN cores proved predictive (Table 2).

In a second large study, $45 \%(n=328)$ underwent further biopsy and $36 \%$ were diagnosed with cancer [15]. A single core of HGPIN was not predictive for a subsequent diagnosis of cancer (HR 1.19, CI 0.87, 1.82, $p=0.41$ ), while two cores or more were (HR 2.56, CI 1.83, 3.60, $p<0.0001$ ). Noteworthy is that patients with two cores positive for HGPIN (HR 2.73) had a higher risk for cancer diagnosis than 3 (HR 2.11) or greater than 4 cores (HR 2.70) which shows the inconsistency of subgroup analysis. Concomitantly, they analyzed a group of patients with a prior benign biopsy and 25\% were diagnosed with cancer on re-biopsy. The benign biopsy patients had a higher PSA $(8.49 \mathrm{ng} / \mathrm{ml})$ than the HGPIN patients $(6.83 \mathrm{ng} / \mathrm{ml})$ so may have just been a higher risk group.

In one final study 262 patients underwent vigorous re-biopsy (20 - 26 cores); $32 \%$ were diagnosed with cancer. [18] It should be noted that a higher number of cores taken initially resulted in fewer cancers at re-biopsy $(<12$ cores initially, $38 \%$ cancer vs $23 \%$ with more than 12 cores, $p=0.01$ ). They found that multiple cores of HGPIN were more predictive $(40 \%)$ than a single core $(25 \%, p=0.013)$. They did not state the PSA for those groups, but patients with a cancer diagnosis had a higher PSA (mean 7.7) than those without (mean 6.6, $p=0.031$ ). On multivariate analysis, they found that PSA, age, and number of cores were predictive for the diagnosis of cancer.

In addition to the institutional studies outlined above, there are several large series based on biopsies sent to reference labs. In one such study, 204 patients

Table 2. HGPIN and predictive factors for subsequent cancer diagnosis.

\begin{tabular}{|c|c|c|c|c|c|c|c|c|c|c|}
\hline \multicolumn{11}{|c|}{ Predictive factors for finding of cancer } \\
\hline Study & $\begin{array}{l}\text { \#HGPIN } \\
\text { patients } \\
\text { re-biopsied }\end{array}$ & $\begin{array}{c}\% \text { of total } \\
\text { HGPIN } \\
\text { patients }\end{array}$ & $\begin{array}{l}\text { Bx time } \\
\text { frame }\end{array}$ & $\begin{array}{l}\text { Percentage } \\
\text { positive for } \\
\text { cancer }\end{array}$ & Age & PSA & PSAD & PSAV & $\begin{array}{l}\text { Number } \\
\text { Cores of } \\
\text { HGPIN }\end{array}$ & $\begin{array}{c}\text { Unilateral } \\
\text { or bilateral } \\
\text { HGPIN }\end{array}$ \\
\hline Herawi [9] & 791 & NS & $\leq 1 \mathrm{yr}$ & $18 \%$ & No & NS & NS & NS & No & NS \\
\hline Lee $[15]$ & 328 & $45 \%$ & $\leq 2.56 \mathrm{yr}$ & $36 \%$ & NS & NS & NS & NS & Yes & Yes \\
\hline Roscigno [18] & 262 & NS & $3-30 \mathrm{mo}$ & $32 \%$ & Yes & Yes & NS & NS & Yes & NS \\
\hline Schlesinger [11] & 204 & NS & $<14 \mathrm{mo}$ & $23 \%$ & NS & NS & NS & NS & NS & NS \\
\hline O’Dowd [16] & 4902 & $27 \%$ & $\leq 1 \mathrm{yr}$ & $23 \%$ & No & Yes & NS & NS & NS & NS \\
\hline Merrimen [12] & 564 & $44 \%$ & $\leq 1.91 \mathrm{yr}$ & $27 \%$ & Yes & NS & NS & NS & Yes & NS \\
\hline Taneja [19] & 1185 & $100 \%$ & $1-3$ years & $32 \%$ & Yes & Yes & NS & Yes & NS & NS \\
\hline Current study & 362 & $64 \%$ & $\begin{array}{c}7 \text { mo } \\
\text { median }\end{array}$ & $37 \%$ & No & Yes & Yes & NS & No & No \\
\hline
\end{tabular}

$\mathrm{Bx}=$ biopsy. $\mathrm{PSA}=$ prostate specific antigen. $\mathrm{PSAD}=$ prostate specific antigen density. PSAV $=$ PSA velocity. Uni $=$ unilateral. $\mathrm{NS}=$ not stated. 
with HGPIN had tissue submitted from a re-biopsy and 23\% (47 patients) were found with cancer [11]. From their review of the literature, they found that the rate had declined from pre-1995 (36\%) to 2002-2003 (21\%). They advocated "that isolated PIN in an extended biopsy warrants repeat biopsy". In another large series from the 1990s, on initial biopsy, 38\% were diagnosed with cancer. Only $4 \%$ were diagnosed with HGPIN $(n=4902), 27 \%$ of whom had another specimen sent in within a year. Twenty three percent of them were found with cancer, which was similar for the patients with benign findings on the first biopsy (20\%) [16]. In a third such study, from 12304 biopsies, initially $47 \%$ were diagnosed with cancer and $10 \%(n=1283)$ were diagnosed with HGPIN [12]. The PSA for those patients was a mean of $7.86( \pm 14.61)$ vs $6.97( \pm 3.63)$ in those that had a benign biopsy. Five hundred sixty-four (44\%) of the HGPIN patients had another biopsy and $27 \%$ were diagnosed with cancer. For the $17 \%$ of the benign biopsy patients that underwent re-biopsy, 22\% were diagnosed with cancer ( $p=$ $0.019)$. Those with a single core of HGPIN had no greater risk of cancer diagnosis (OR 1.02) than those with benign disease. Those with multiple cores did, increasing with each additional core until 5 and then the OR decreased and the $p$ value became non-significant, again indicating the danger of retrospective data and diminishing numbers. It is noteworthy that those with multiple cores of high grade PIN also had a higher PSA $(8.86 \mathrm{ng} / \mathrm{ml})$ than those with a single core $(6.47 \mathrm{ng} / \mathrm{ml}$ ), which raises the issue that other factors (other than the number of HGPIN positive cores) may have contributed. None the less, they stated: "Results suggest that follow up should be more rigorous in patients with multifocal PIN. We recommend that patients with multifocal HGPIN undergo repeat biopsy within 1 year." (page 489). As seen in Table 2, the literature is inconsistent and a review of multiple studies showed that the number of cores positive for HGPIN was not predictive [3].

Many retrospective reports call for prospective studies to help resolve the issue, but those are rarely done. There is one prospective study in HGPIN patients. While the goal of the study was the prevention of subsequent prostate cancer, since all the patients were mandated for re-biopsy, the results are informative.

Toremifene citrate is an estrogen receptor modulator used in breast cancer. Some early studies suggested that it might modulate the development of prostate cancer. To that end, a randomized study was undertaken in HGPIN patients (with at least a 10 core biopsy and PSA $<10 \mathrm{ng} / \mathrm{ml}$ ) [19]. Nineteen percent also had atypical small acinar proliferation, which was found to be a significant predictor for cancer (HR 1.91) on multivariate analysis, but were not excluded from analysis. Biopsies were required at 12, 24 and 36 months. Toremifene citrate did not change the rate of cancer diagnosis. Of the patients that completed the study, $32 \%(378 / 1185)$ were diagnosed with prostate cancer. The positive biopsy rate at year 1 was $17.9 \%$, year 2 was $12.9 \%$ and year 3 was $13.6 \%$. Interestingly, $60 \%$ of the patients with a single core of HGPIN were free of cancer at 3 years vs $51 \%$ ( $p$ 
$<0.001)$ with more than one core, but this dropped out as a significant finding on multivariate analysis. At least in this study, the finding of multiple cores was not an independent predictive factor. Overall, it is likely these patients represent a somewhat lower risk sampling (PSA $<10 \mathrm{ng} / \mathrm{ml}$ ) of HGPIN patients, but the $32 \%$ risk of prostate cancer should be a fairly reliable benchmark-it certainly is consistent with the larger retrospective studies discussed above.

After 20 years of discussion, the exact significance of HGPIN remains elusive. It has been argued that in the era of increased (10+) biopsy cores that the finding of HGPIN should be considered a benign finding [3]. Others still argue that HGPIN is a precursor for prostate cancer (apparently with very slow or incomplete penetrance), or at the least a marker for prostate cancer [2]. In most studies, HGPIN patients with a subsequent diagnosis of prostate cancer, on the average, have a higher PSA than those who don't, demonstrating that there is at least one confounding factor for the diagnosis of cancer.

Given that the re-biopsy rate in the literature is below $50 \%$, it is clear that the finding of HGPIN alone is not mandating a re-biopsy. Urologists are already considering other factors (age, comorbidities, PSA level) to determine whether another biopsy is indicated.

The overall incidence of cancer on biopsy after a finding of high grade PIN is $\sim 30 \%$. This is significantly lower than that of initial biopsy (47\% [12], 38\% [16]). In studies that have a comparative cohort with benign biopsy undergoing re-biopsy, there is not a great difference in the positive cancer rate between those patients (22\% [12], 20\% [16]) and patients re-biopsied after a diagnosis of high grade PIN (27\% [12], 23\% [16]). Altogether, it does not appear an isolated finding of high grade PIN is a strong predictor of subsequent diagnosis of cancer and would appear only to be a relative risk. For patients with high grade PIN, other than a slightly higher awareness, we are left with our imperfect standard indicators for re-biopsy, such as absolute PSA, change in PSA (including velocity), PSA density, symptoms, and physical exam weighed against the patient's age and comorbidities. Until better markers are discovered, this is the current "state of the art" with HGPIN.

\section{References}

[1] Bostwick, D.G. (1996) Prospective Origins of Prostate Carcinoma. Prostatic Intraepithelial Neoplasia and Atypical Adenomatous Hyperplasia. Cancer, 78, 330-336. https://doi.org/10.1002/(SICI)1097-0142(19960715)78:2<330::AID-CNCR22>3.0.C $\mathrm{O} ; 2-\mathrm{W}$

[2] Bostwick, D.G., Liu, L., Brawer, M.K. and Qian, J. (2004) High-Grade Prostatic Intraepithelial Neoplasia. Reviews in Urology, 6, 171-179. https://doi.org/10.1038/modpathol.3800053

[3] Epstein, J.I. and Herawi, M. (2006) Prostate Needle Biopsies Containing Prostatic Intraepithelial Neoplasia or Atypical Foci Suspicious for Carcinoma: Implications for Patient Care. Journal of Urology, 175, 820-834. https://doi.org/10.1016/S0022-5347(05)00337-X

[4] Epstein, J.I. and Potter, S.R. (2001) The Pathological Interpretation and Significance 
of Prostate Needle Biopsy Findings: Implications and Current Controversies. Journal of Urology, 166, 402-410. https://doi.org/10.1016/S0022-5347(05)65953-8

[5] Naya, Y., Ayala, A., Tamboli, P. and Babaian, R. (2004) Can the Number of Cores with High-Grade Prostate Intraepithelial Neoplasia Predict Cancer in Men Who Undergo Repeat Biopsy? Urology, 63, 503-508.

https://doi.org/10.1016/j.urology.2003.09.066

[6] Raviz, G., Ziotta, A., Janssen, Th., et al. (1996) Do Prostate Specific Antigen and Prostate Specific Antigen Density Enhance the Detection of Prostate Carcinoma After Initial Diagnosis of Prostatic Intraepithelial Neoplasia without Concurrent Carcinoma? Cancer, 77, 2103-2108.

https://doi.org/10.1002/(SICI)1097-0142(19960515)77:10<2103::AID-CNCR21>3.0. CO;2-Y

[7] Bishara, T., Ramnani, D.M. and Epstein, J.I. (2004) High-Grade Prostatic Intraepithelial Neoplasia on Needle Biopsy: Risk of Cancer on Repeat biopsy Related to Number of Involved Cores and Morphologic Pattern. The American Journal of Surgical Pathology, 28, 629-633. https://doi.org/10.1097/00000478-200405000-00010

[8] Davidson, D., Bostwick, D.G., Qian, J., et al. (1995) Prostatic Intraepithelial Neoplasia Is a Risk Factor for Adenocarcinoma: Predictive Accuracy in Needle Biopsies. Journal of Urology, 154, 1295-1299. https://doi.org/10.1016/S0022-5347(01)66840-X

[9] Herawi, M., Kahane, H., Epstein, J.I., et al. (2006) Risk of Prostate Cancer on First Re-Biopsy within 1 Year Following a Diagnosis of High Grade Prostatic Intraepithelial Neoplasia Is Related to the Number of Cores Sampled. Journal of Urology, 175, 121-124. https://doi.org/10.1016/S0022-5347(05)00064-9

[10] Netto, G.J. and Epstein, J.I. (2006) Widespread High-Grade Prostatic Intraepithelial Neoplasia on Prostatic Needle Biopsy: A Significant Likelihood of Subsequently Diagnosed Adenocarcinoma. The American Journal of Surgical Pathology, 30, 1184-1188. https://doi.org/10.1097/01.pas.0000213324.97294.54

[11] Schlesinger, C., Bostwick, D.G. and Iczkowski, K.A. (2005) High-Grade Prostatic Intraepithelial Neoplasia and Atypical Small Acinar Proliferation: Predictive Value for Cancer in Current Practice. The American Journal of Surgical Pathology, 29, 1201-1207. https://doi.org/10.1097/01.pas.0000168178.48535.0d

[12] Merrimen, J.L., Jones, G., Walker, D., Leung, C.S., Kapusta, L.R. and Srigley, J.R. (2009) Multifocal High Grade Prostatic Intraepithelial Neoplasia Is a Significant Risk Factor for Prostatic Adenocarcinoma. Journal of Urology, 182, 485-490. https://doi.org/10.1016/j.juro.2009.04.016

[13] Presti, J.C. (2003) Prostate Biopsy: How Many Cores Are Enough? Urologic Oncology, 21, 135-140. https://doi.org/10.1016/S1078-1439(03)00006-1

[14] European Association of Urology (2015) Guidelines on Prostate Cancer. https://uroweb.org/wp-content/uploads/09-Prostate-Cancer_LR.pdf

[15] Lee, M.C., Moussa, A.S., Yu, C., Kattan, M.W., Magi-Galluzzi, C. and Jones, J.S. (2010) Multifocal High Grade Prostatic Intraepithelial Neoplasia Is a Risk Factor for Subsequent Prostate Cancer. Journal of Urology, 184, 1958-1962. https://doi.org/10.1016/j.juro.2010.06.137

[16] O’Dowd, G.J., Miller, M.C., Orozco, R. and Veltri, R.W. (2000) Analysis of Repeated Biopsy Results within 1 Year after a Noncancer Diagnosis. Urology, 55, 553-559. https://doi.org/10.1016/S0090-4295(00)00447-7

[17] Kronz, J.D., Allan, C.H., Shaikh, A.A. and Epstein, J.I. (2001) Predicting Cancer Following a Diagnosis of High-Grade Prostatic Intraepithelial Neoplasia on Needle 
Biopsy: Data on Men with More than One Follow-Up Biopsy. The American Journal of Surgical Pathology, 25, 1079-1085.

https://doi.org/10.1097/00000478-200108000-00014

[18] Roscigno, M., Scattoni, V., Freschi, M., et al. (2012) Diagnosis of Isolated High-Grade Prostatic Intra-Epithelial Neoplasia: Proposal of a Nomogram for the Prediction of Cancer Detection at Saturation Re-Biopsy. BJU International, 109, 1329-1334. https://doi.org/10.1111/j.1464-410X.2011.10532.x

[19] Taneja, S.S., Morton, R., Barnette, G., Sieber, P., Hancock, M.L. and Steiner, M. (2013) Prostate Cancer Diagnosis among Men with Isolated High-Grade Intraepithelial Neoplasia Enrolled onto a 3-Year Prospective Phase III Clinical Trial of Oral Toremifene. Journal of Clinical Oncology, 31, 523-529.

https://doi.org/10.1200/JCO.2012.41.7634 